Supporting Information for

\title{
Hetero-metallic active sites in omega (MAZ) zeolite catalyzed methane partial oxidation: a DFT study
}

Linzhe Wang a , Zhi Li ${ }^{\text {a }}$, Zhixia Wang a , Xinyu Chen a, Weiyu Song a,*, Zhen Zhao ${ }^{\text {a }}$, Yuechang Wei ${ }^{\text {a }}$, Xiao Zhang ${ }^{\text {a }}$

${ }^{a}$ State Key Laboratory of Heavy Oil Processing, College of Science, China University of Petroleum-Beijing, Beijing 102249, China

Figure S1 The optimized structures of $[\mathrm{Cu}-\mathrm{O}-\mathrm{M}]^{2+}(\mathrm{M}=\mathrm{Pd}, \mathrm{Pt}, \mathrm{Fe}, \mathrm{Co}, \mathrm{Ni}, \mathrm{Au}$, $\mathrm{Ag}$ ) in low-spin and high-spin states

Table S1 The relative energies of $[\mathrm{Cu}-\mathrm{O}-\mathrm{M}]^{2+}(\mathrm{M}=\mathrm{Pd}, \mathrm{Pt}, \mathrm{Fe}, \mathrm{Co}, \mathrm{Ni}, \mathrm{Au}, \mathrm{Ag})$ in low-spin and high-spin states

Figure S2 The $\mu-\mathrm{O}$ p-projected density of states in $[\mathrm{Cu}-\mathrm{O}-\mathrm{M}]^{2+}(\mathrm{M}=\mathrm{Pd}, \mathrm{Pt}, \mathrm{Fe}$, $\mathrm{Co}, \mathrm{Ni}, \mathrm{Au})$

Table S2 The key structural parameters of RC, TS1, RI, TS2 and PC of MTM reaction in $[\mathrm{Cu}-\mathrm{O}-\mathrm{M}]^{2+}-\mathrm{MAZ}(\mathrm{M}=\mathrm{Pd}, \mathrm{Pt}, \mathrm{Fe}, \mathrm{Co}, \mathrm{Ni}, \mathrm{Au}, \mathrm{Ag})$

Figures Potential energy diagrams of $[\mathrm{Cu}-\mathrm{O}-\mathrm{M}]^{2+}$ in low-spin and high-spin S3-S9 states

Figure S10 The atom spin density of TS1 (Isosurface value is $0.015 \mathrm{eV} / \AA^{3}$ )

Figure S11 The C p-projected density of states in TS1 of $[\mathrm{Cu}-\mathrm{O}-\mathrm{M}]^{2+}(\mathrm{M}=\mathrm{Pd}, \mathrm{Pt}$, $\mathrm{Fe}, \mathrm{Co}, \mathrm{Ni}, \mathrm{Au}, \mathrm{Ag})$

Table S3 The oxidation reaction energies $\left(E_{\mathrm{r} 3}\right)$ and energy barriers $\left(E_{\mathrm{a}}^{\mathrm{TS} 3}\right)$ of reduced $[\mathrm{Cu}-\mathrm{M}]^{2+}$ to $[\mathrm{Cu}-\mathrm{O}-\mathrm{M}]^{2+}(\mathrm{M}=\mathrm{Pd}, \mathrm{Pt}, \mathrm{Fe}, \mathrm{Co}, \mathrm{Ni}, \mathrm{Au}, \mathrm{Ag})$ active site regeneration with $\mathrm{N}_{2} \mathrm{O}$ oxidant

Figure S12 The linear relationship between $\angle \mathrm{Cu}-\mathrm{O}-\mathrm{M}(\mathrm{M}=\mathrm{Pd}, \mathrm{Pt}, \mathrm{Fe}, \mathrm{Co}, \mathrm{Ni}$, $\mathrm{Au}, \mathrm{Ag})$ of $[\mathrm{Cu}-\mathrm{O}-\mathrm{M}]^{2+}$ and energy barrier of methane activation 

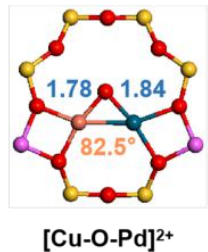

$(\mathrm{SM}=2)$

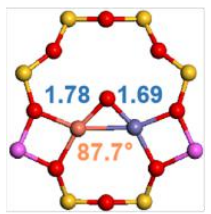

[Cu-O-Fe] ${ }^{2+}$ $(\mathrm{SM}=4)$

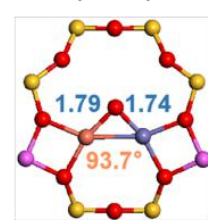

[Cu-O-Fe] ${ }^{2+}$ $(\mathrm{SM}=6)$

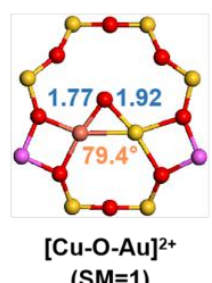

$(\mathrm{SM}=1)$

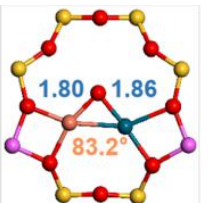

[Cu-O-Pd] ${ }^{2+}$

$(\mathrm{SM}=4)$

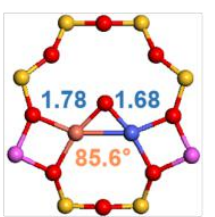

[Cu-O-Co] ${ }^{2+}$

$(\mathrm{SM}=3)$

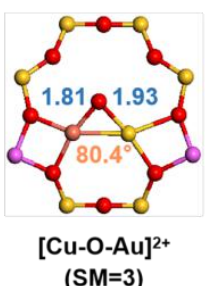

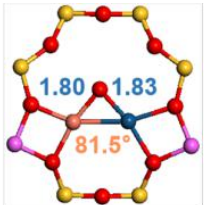

[Cu-O-Pt] ${ }^{2+}$

(SM=2)

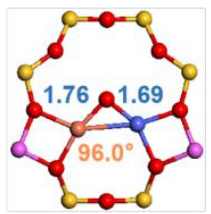

[Cu-O-Co] ${ }^{2+}$

$(\mathrm{SM}=5)$

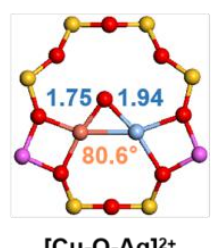

[Cu-O-Ag] ${ }^{2+}$

$(\mathrm{SM}=1)$

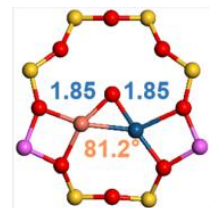

[Cu-O-Pt] ${ }^{2+}$

$(\mathrm{SM}=4)$

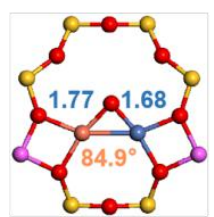

[Cu-O-Ni] ${ }^{2+}$

$(\mathrm{SM}=2)$

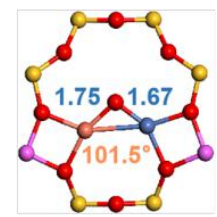

[Cu-O-Ni] ${ }^{2+}$

$(\mathrm{SM}=4)$

Figure S1. The optimized structures of $[\mathrm{Cu}-\mathrm{O}-\mathrm{M}]^{2+}(\mathrm{M}=\mathrm{Pd}, \mathrm{Pt}, \mathrm{Fe}, \mathrm{Co}, \mathrm{Ni}, \mathrm{Au}, \mathrm{Ag})$ in low-spin and high-spin state, in which the left and right blue numbers represent bond length of $\mathrm{Cu}-\mathrm{O}$ and $\mathrm{M}-\mathrm{O}$ respectively and the dark yellow numbers stands for bond angle $\angle \mathrm{Cu}-\mathrm{O}-\mathrm{M}$.

Table $\mathbf{S 1}$ The relative energies of $[\mathrm{Cu}-\mathrm{O}-\mathrm{M}]^{2+}(\mathrm{M}=\mathrm{Pd}, \mathrm{Pt}, \mathrm{Fe}, \mathrm{Co}, \mathrm{Ni}, \mathrm{Au}, \mathrm{Ag})$

\begin{tabular}{ccccc}
\hline$[\mathrm{Cu}-\mathrm{O}-\mathrm{M}]^{2+}$ & $\begin{array}{c}\text { spin } \\
\text { multiplicity }\end{array}$ & $\begin{array}{c}\text { relative } \\
\text { energy /eV }\end{array}$ & $\begin{array}{c}\text { spin } \\
\text { multiplicity }\end{array}$ & $\begin{array}{c}\text { relative } \\
\text { energy /eV }\end{array}$ \\
\hline$[\mathrm{Cu}-\mathrm{O}-\mathrm{Pd}]^{2+}$ & 2 & 0.00 & 4 & 1.01 \\
{$[\mathrm{Cu}-\mathrm{O}-\mathrm{Pt}]^{2+}$} & 2 & 0.00 & 4 & 1.28 \\
{$[\mathrm{Cu}-\mathrm{O}-\mathrm{Fe}]^{2+}$} & 4 & 0.00 & 6 & 0.52 \\
{$[\mathrm{Cu}-\mathrm{O}-\mathrm{Co}]^{2+}$} & 3 & 0.00 & 5 & 0.23 \\
{$[\mathrm{Cu}-\mathrm{O}-\mathrm{Ni}]^{2+}$} & 2 & 0.00 & 4 & 0.50 \\
{$[\mathrm{Cu}-\mathrm{O}-\mathrm{Au}]^{2+}$} & 1 & 0.00 & 3 & 0.43 \\
{$[\mathrm{Cu}-\mathrm{O}-\mathrm{Ag}]^{2+}$} & 1 & 0.00 & 3 & 0.12 \\
\hline
\end{tabular}



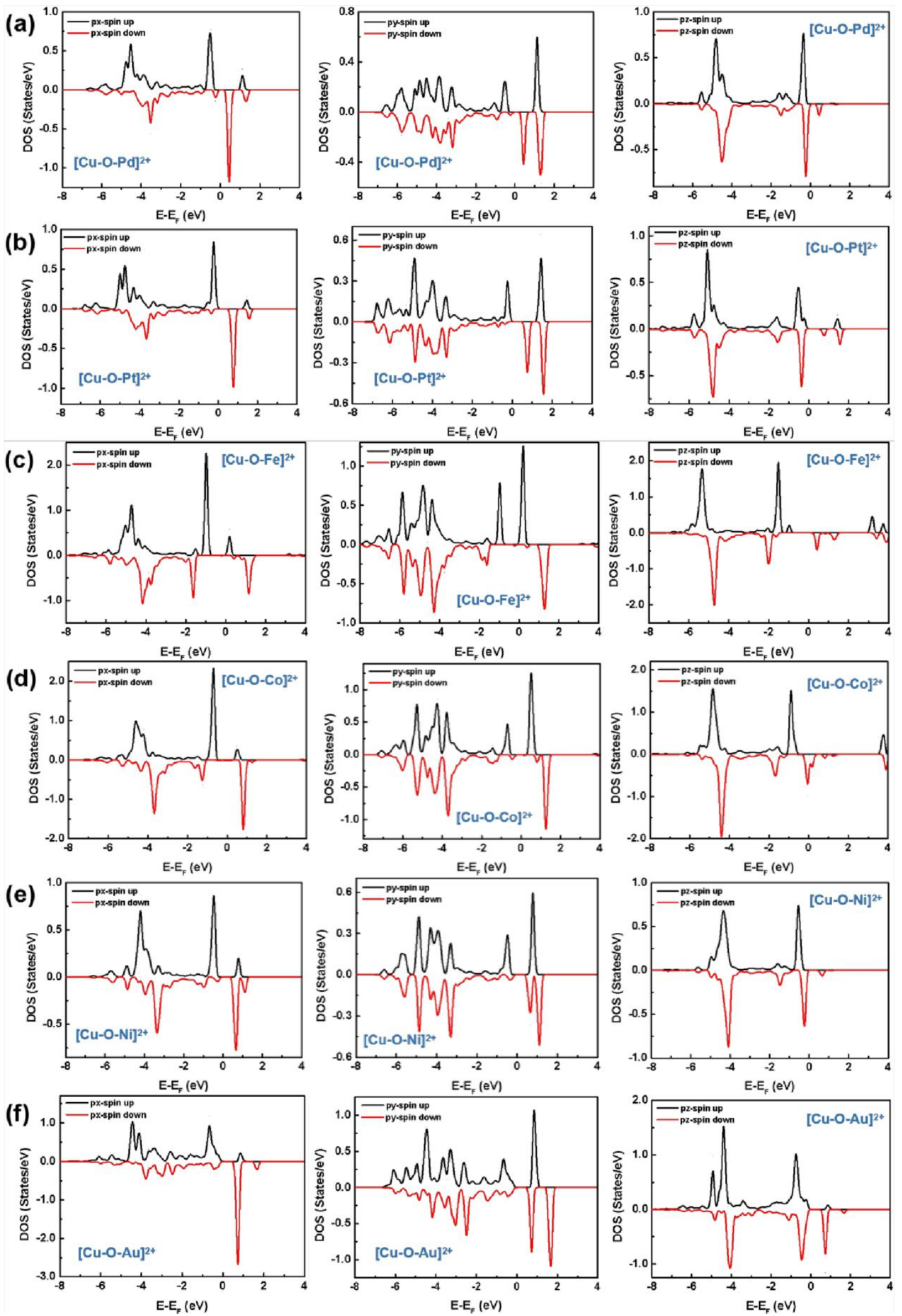

Figure S2. The $\mu-\mathrm{O}$ p-projected density of states in $[\mathrm{Cu}-\mathrm{O}-\mathrm{M}]^{2+}(\mathrm{M}=\mathrm{Pd}, \mathrm{Pt}, \mathrm{Fe}, \mathrm{Co}, \mathrm{Ni}, \mathrm{Au})$.

The three plots in each $[\mathrm{Cu}-\mathrm{O}-\mathrm{M}]^{2+}$ represent p-projected density of states of $\mathrm{p}_{\mathrm{x}}, \mathrm{p}_{\mathrm{y}}$ and $\mathrm{p}_{z}$ respectively. 
Table S2 The key structural parameters of RC, TS1, RI, TS2 and PC of MTM reaction in $[\mathrm{Cu}-\mathrm{O}-\mathrm{M}]^{2+}-\mathrm{MAZ}(\mathrm{M}=\mathrm{Pd}, \mathrm{Pt}, \mathrm{Fe}, \mathrm{Co}, \mathrm{Ni}, \mathrm{Au}, \mathrm{Ag})$

\begin{tabular}{|c|c|c|c|c|c|}
\hline$[\mathrm{Cu}-\mathrm{O}-\mathrm{Pd}]^{2+}$ & $\angle \mathrm{Cu}-\mathrm{O}-\mathrm{Pd} / \mathrm{deg}$ & $\mathrm{d}_{(\mathrm{Cu}-\mathrm{O})} / \AA$ & $\mathrm{d}_{(\mathrm{M}-\mathrm{O})} / \AA$ & $\mathrm{d}_{(\mathrm{C}-\mathrm{H})} / \AA$ & $\mathrm{d}_{(\mathrm{O}-\mathrm{H})} / \AA$ \\
\hline $\mathrm{RC}$ & 82.8 & 1.78 & 1.84 & 1.10 & 2.90 \\
\hline TS1 & 79.3 & 1.83 & 1.95 & 2.39 & 0.99 \\
\hline RI & 78.7 & 1.84 & 1.96 & 3.81 & 0.98 \\
\hline TS2 & 79.6 & 1.83 & 1.95 & 3.29 & 0.98 \\
\hline $\mathrm{PC}$ & 70.7 & 2.02 & 2.18 & 2.05 & 0.99 \\
\hline$[\mathrm{Cu}-\mathrm{O}-\mathrm{Pt}]^{2+}$ & $\angle \mathrm{Cu}-\mathrm{O}-\mathrm{Pt} / \mathrm{deg}$ & $\mathrm{d}_{(\mathrm{Cu}-\mathrm{O})} / \AA$ & $\mathrm{d}_{(\mathrm{M}-\mathrm{O})} / \AA$ & $\mathrm{d}_{(\mathrm{C}-\mathrm{H})} / \AA$ & $\mathrm{d}_{(\mathrm{O}-\mathrm{H})} / \AA$ \\
\hline $\mathrm{RC}$ & 81.8 & 1.80 & 1.83 & 1.10 & 3.29 \\
\hline TS1 & 78.5 & 1.84 & 1.95 & 3.19 & 0.98 \\
\hline RI & 77.7 & 1.85 & 1.96 & 4.21 & 0.98 \\
\hline TS2 & 75.7 & 1.90 & 1.99 & 2.91 & 0.99 \\
\hline $\mathrm{PC}$ & 60.3 & 2.54 & 2.05 & 2.05 & 0.99 \\
\hline$[\mathrm{Cu}-\mathrm{O}-\mathrm{Fe}]^{2+}$ & $\angle \mathrm{Cu}-\mathrm{O}-\mathrm{Fe} / \mathrm{deg}$ & $\mathrm{d}_{(\mathrm{Cu}-\mathrm{O})} / \AA$ & $\mathrm{d}_{(\mathrm{M}-\mathrm{O})} / \AA$ & $\mathrm{d}_{(\mathrm{C}-\mathrm{H})} / \AA$ & $\mathrm{d}_{(\mathrm{O}-\mathrm{H})} / \AA$ \\
\hline $\mathrm{RC}$ & 87.6 & 1.78 & 1.69 & 1.10 & 3.39 \\
\hline $\mathrm{TS} 1$ & 80.4 & 1.86 & 1.86 & 2.82 & 0.98 \\
\hline $\mathrm{RI}$ & 80.2 & 1.86 & 1.86 & 4.45 & 0.98 \\
\hline TS2 & 58.8 & 2.27 & 2.36 & 2.02 & 0.98 \\
\hline $\mathrm{PC}$ & 53.2 & 2.86 & 1.96 & 2.04 & 0.99 \\
\hline$[\mathrm{Cu}-\mathrm{O}-\mathrm{Co}]^{2+}$ & $\angle \mathrm{Cu}-\mathrm{O}-\mathrm{Co} / \mathrm{deg}$ & $\mathrm{d}_{(\mathrm{Cu}-\mathrm{O})} / \AA$ & $\mathrm{d}_{(\mathrm{M}-\mathrm{O})} / \AA$ & $\mathrm{d}_{(\mathrm{C}-\mathrm{H})} / \AA$ & $\mathrm{d}_{(\mathrm{O}-\mathrm{H})} / \AA$ \\
\hline $\mathrm{RC}$ & 85.6 & 1.78 & 1.68 & 1.10 & 3.02 \\
\hline TS1 & 82.2 & 1.84 & 1.80 & 2.80 & 0.98 \\
\hline RI & 81.2 & 1.86 & 1.83 & 4.27 & 0.98 \\
\hline TS2 & 73.0 & 1.94 & 2.00 & 2.36 & 0.99 \\
\hline $\mathrm{PC}$ & 54.5 & 2.78 & 1.90 & 2.05 & 0.98 \\
\hline$[\mathrm{Cu}-\mathrm{O}-\mathrm{Ni}]^{2+}$ & $\angle \mathrm{Cu}-\mathrm{O}-\mathrm{Ni} / \mathrm{deg}$ & $\mathrm{d}_{(\mathrm{Cu}-\mathrm{O})} / \AA$ & $\mathrm{d}_{(\mathrm{M}-\mathrm{O})} / \AA$ & $\mathrm{d}_{(\mathrm{C}-\mathrm{H})} / \AA$ & $\mathrm{d}_{(\mathrm{O}-\mathrm{H})} / \AA$ \\
\hline $\mathrm{RC}$ & 84.9 & 1.77 & 1.68 & 1.10 & 3.05 \\
\hline $\mathrm{TS} 1$ & 85.6 & 1.81 & 1.77 & 3.85 & 0.98 \\
\hline RI & 81.1 & 1.83 & 1.80 & 4.04 & 0.98 \\
\hline $\mathrm{TS} 2$ & 80.4 & 1.85 & 1.81 & 3.56 & 0.98 \\
\hline $\mathrm{PC}$ & 70.1 & 2.13 & 1.95 & 2.04 & 0.99 \\
\hline$[\mathrm{Cu}-\mathrm{O}-\mathrm{Au}]^{2+}$ & $\angle \mathrm{Cu}-\mathrm{O}-\mathrm{Au} / \mathrm{deg}$ & $\mathrm{d}_{(\mathrm{Cu}-\mathrm{O})} / \AA$ & $\mathrm{d}_{(\mathrm{M}-\mathrm{O})} / \AA$ & $\mathrm{d}_{(\mathrm{C}-\mathrm{H})} / \AA$ & $\mathrm{d}_{(\mathrm{O}-\mathrm{H})} / \AA$ \\
\hline $\mathrm{RC}$ & 79.4 & 1.77 & 1.92 & 1.10 & 3.09 \\
\hline TS1 & 76.9 & 1.88 & 2.00 & 3.16 & 0.98 \\
\hline RI & 75.9 & 1.89 & 2.02 & 4.30 & 0.98 \\
\hline TS2 & 71.5 & 1.93 & 2.18 & 2.51 & 0.99 \\
\hline
\end{tabular}




\begin{tabular}{cccccc}
$\mathrm{PC}$ & 69.7 & 1.91 & 2.37 & 2.09 & 1.01 \\
\hline$[\mathrm{Cu}-\mathrm{O}-\mathrm{Ag}]^{2+}$ & $\angle \mathrm{Cu}-\mathrm{O}-\mathrm{Ag} / \mathrm{deg}$ & $\mathrm{d}_{(\mathrm{Cu}-\mathrm{O})} / \AA$ & $\mathrm{d}_{(\mathrm{M}-\mathrm{O})} / \AA$ & $\mathrm{d}_{(\mathrm{C}-\mathrm{H})} / \AA$ & $\mathrm{d}_{(\mathrm{O}-\mathrm{H})} / \AA$ \\
\hline $\mathrm{RC}$ & 80.7 & 1.75 & 1.94 & 1.10 & 3.18 \\
$\mathrm{TS} 1$ & 78.8 & 1.84 & 2.04 & 3.09 & 0.98 \\
$\mathrm{RI}$ & 77.5 & 1.84 & 2.07 & 4.58 & 0.98 \\
$\mathrm{TS} 2$ & 79.4 & 1.82 & 2.07 & 3.10 & 0.97 \\
$\mathrm{PC}$ & 69.2 & 1.92 & 2.43 & 2.04 & 0.98 \\
\hline
\end{tabular}

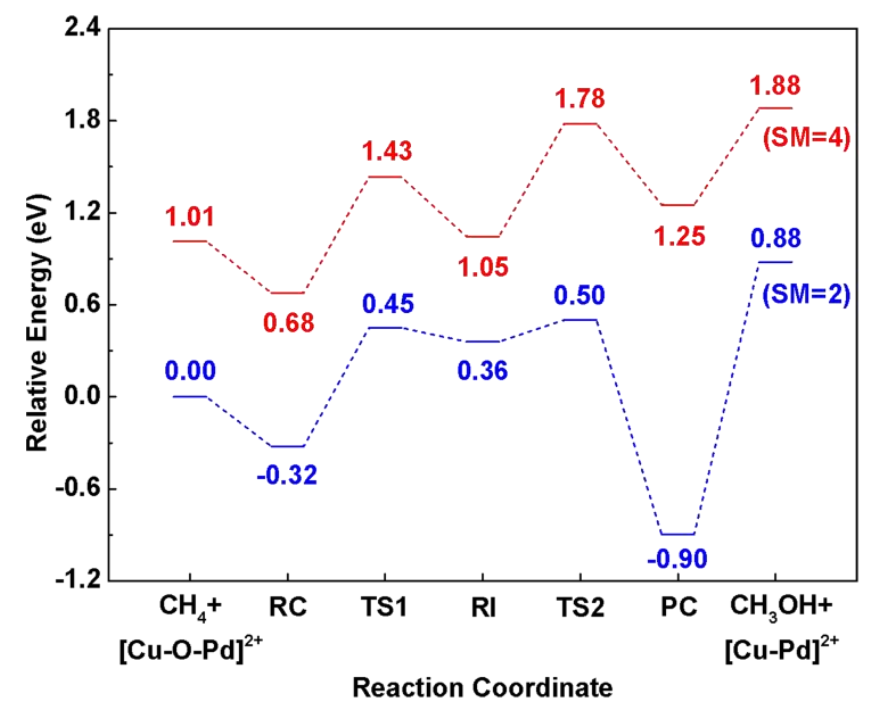

Figure S3. Potential energy diagrams of $[\mathrm{Cu}-\mathrm{O}-\mathrm{Pd}]^{2+}$ in low-spin and high-spin states.

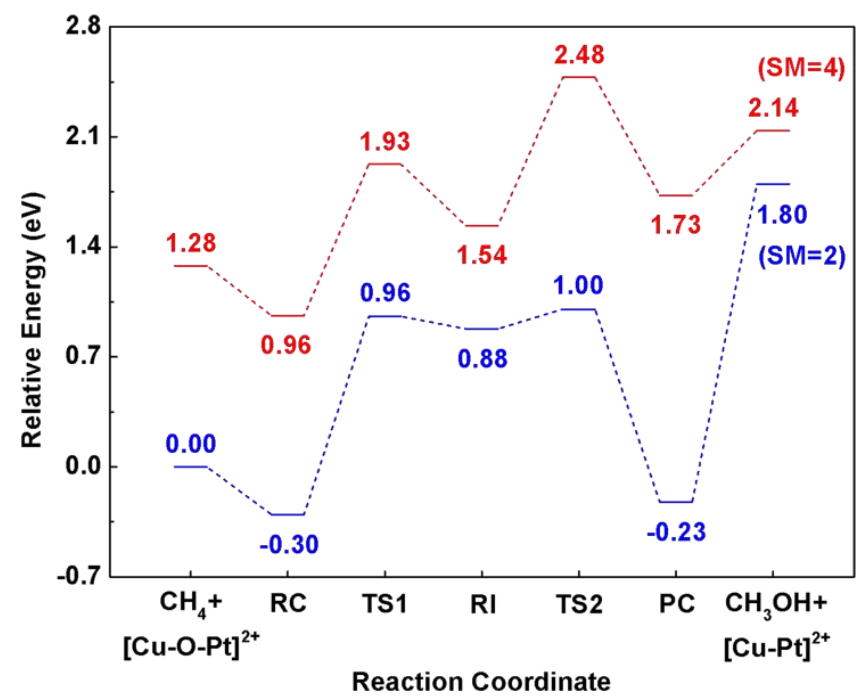

Figure S4. Potential energy diagrams of $[\mathrm{Cu}-\mathrm{O}-\mathrm{Pt}]^{2+}$ in low-spin and high-spin states. 


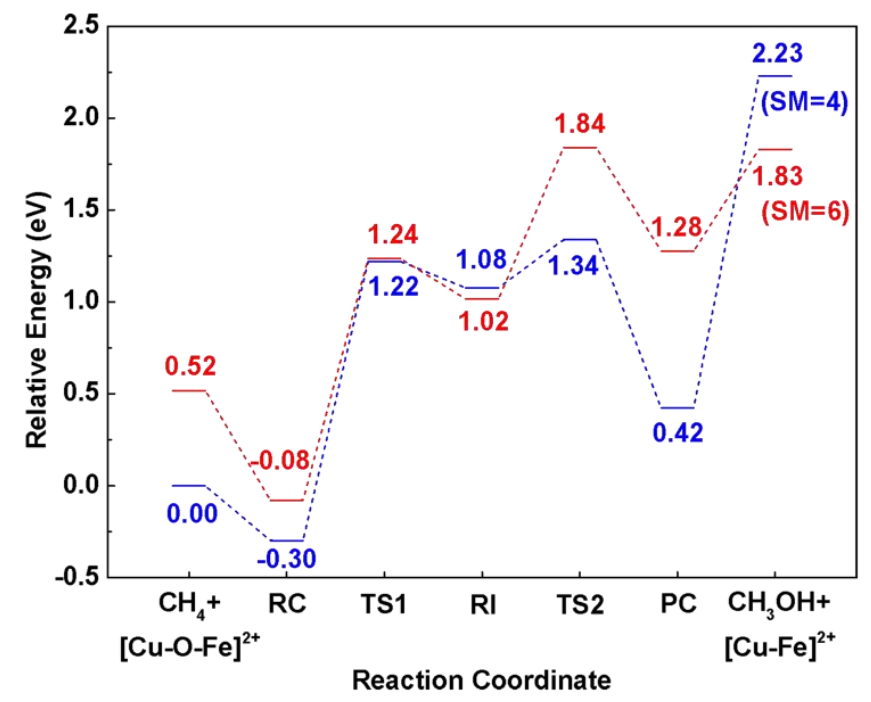

Figure S5. Potential energy diagrams of $[\mathrm{Cu}-\mathrm{O}-\mathrm{Fe}]^{2+}$ in low-spin and high-spin states.

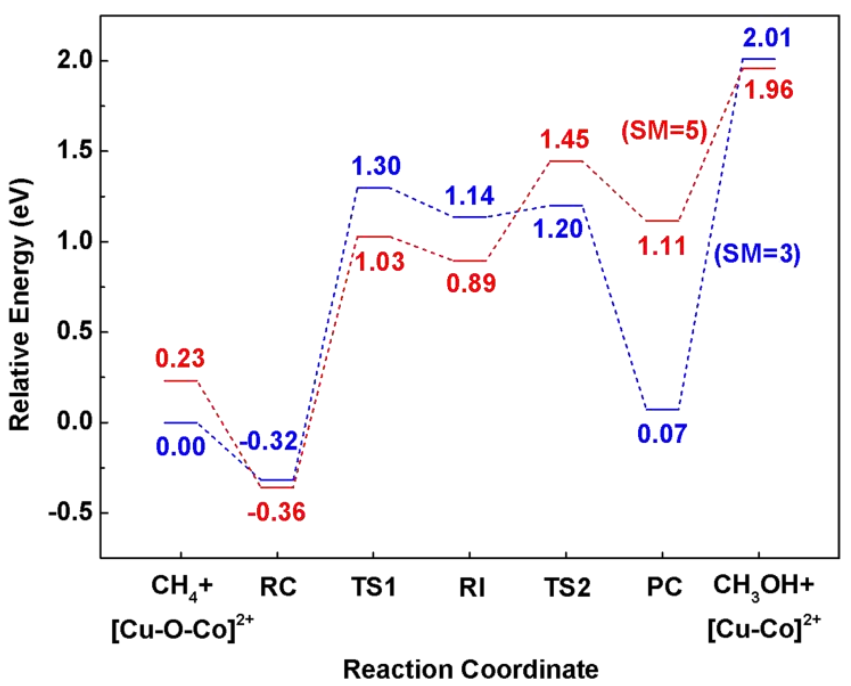

Figure S6. Potential energy diagrams of $[\mathrm{Cu}-\mathrm{O}-\mathrm{Co}]^{2+}$ in low-spin and high-spin states. 


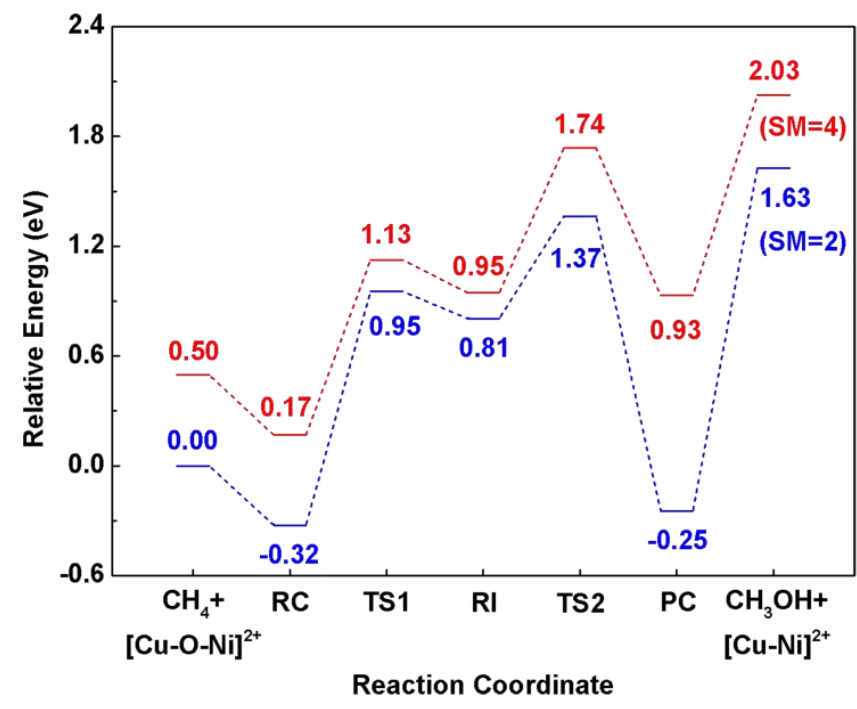

Figure S7. Potential energy diagrams of $[\mathrm{Cu}-\mathrm{O}-\mathrm{Ni}]^{2+}$ in low-spin and high-spin states.

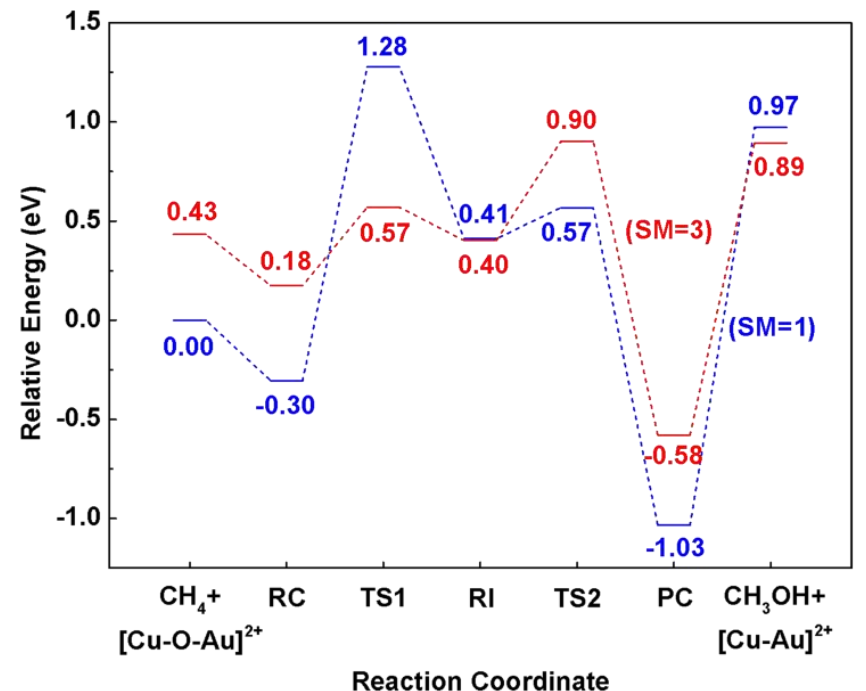

Figure S8. Potential energy diagrams of $[\mathrm{Cu}-\mathrm{O}-\mathrm{Au}]^{2+}$ in low-spin and high-spin states. 


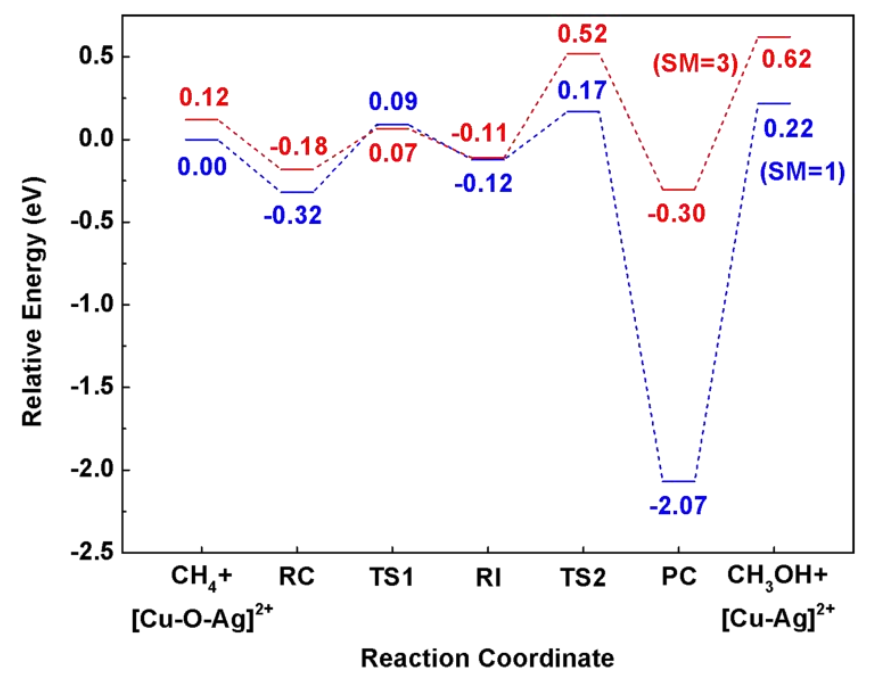

Figure S9. Potential energy diagrams of $[\mathrm{Cu}-\mathrm{O}-\mathrm{Ag}]^{2+}$ in low-spin and high-spin states.

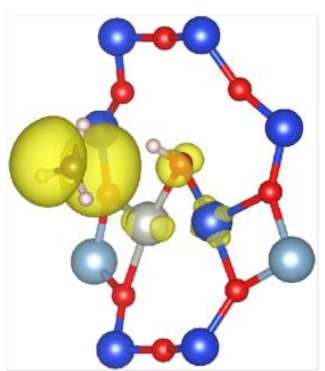

$[\mathrm{Cu}-\mathrm{O}-\mathrm{Pd}]^{2+}$

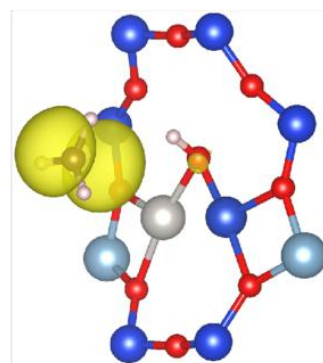

[Cu-O-Pt] $]^{2+}$

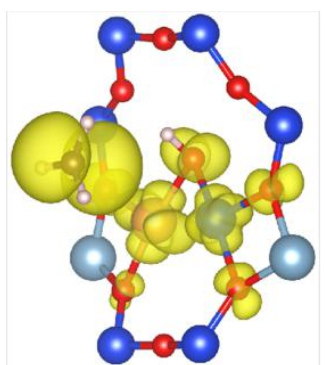

$[\mathrm{Cu}-\mathrm{O}-\mathrm{Au}]^{2+}$

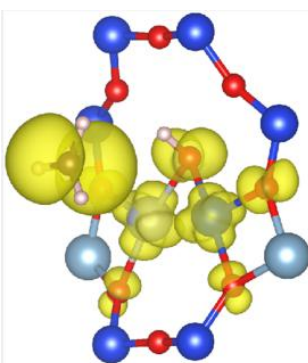

$[\mathrm{Cu}-\mathrm{O}-\mathrm{Ag}]^{2+}$

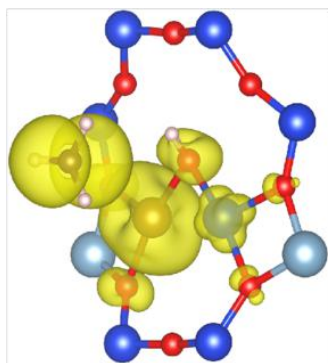

[Cu-O-Fe] ${ }^{2+}$

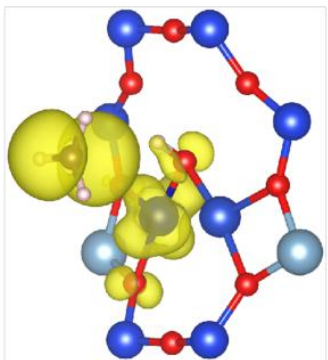

[Cu-O-Co $]^{2+}$

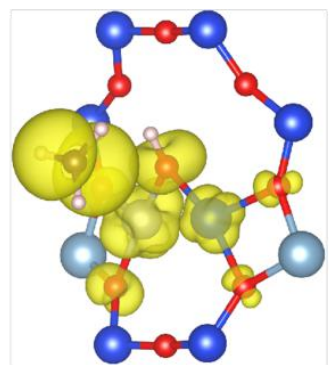

$[\mathrm{Cu}-\mathrm{O}-\mathrm{Ni}]^{2+}$

Figure S10. The atom spin density of TS1 (Isosurface value is $0.015 \mathrm{eV} / \AA^{3}$. Yellow and blue isosurfaces represent positive and negative spin densities respectively.). 

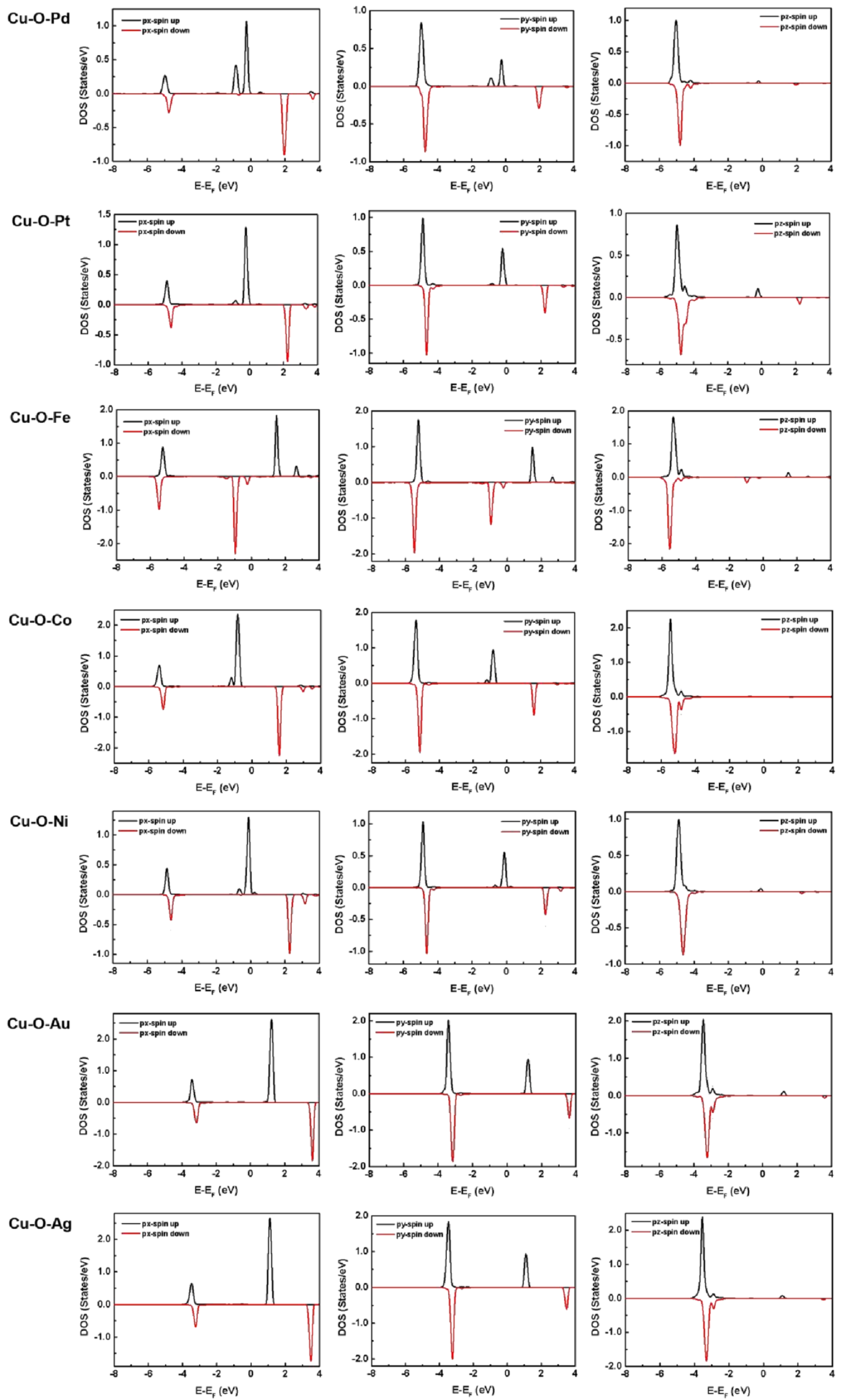
Figure S11. The C p-projected density of states in TS1 of $[\mathrm{Cu}-\mathrm{O}-\mathrm{M}]^{2+}(\mathrm{M}=\mathrm{Pd}, \mathrm{Pt}, \mathrm{Fe}, \mathrm{Co}, \mathrm{Ni}$, $\mathrm{Au}, \mathrm{Ag})$. The three plots in each $[\mathrm{Cu}-\mathrm{O}-\mathrm{M}]^{2+}$ represent p-projected density of states of px, py and pz respectively.

Table S3 The oxidation reaction energies $\left(E_{\mathrm{r} 3}\right)$ and energy barriers $\left(E_{\mathrm{a}}^{\mathrm{TS} 3}\right)$ of reduced $[\mathrm{Cu}-\mathrm{M}]^{2+}$ to $[\mathrm{Cu}-\mathrm{O}-\mathrm{M}]^{2+}(\mathrm{M}=\mathrm{Pd}, \mathrm{Pt}, \mathrm{Fe}, \mathrm{Co}, \mathrm{Ni}, \mathrm{Au}, \mathrm{Ag})$ active site regeneration with $\mathrm{N}_{2} \mathrm{O}$ oxidant

\begin{tabular}{lll}
\hline$[\mathrm{Cu}-\mathrm{O}-\mathrm{M}]^{2+}$ & $E_{\mathrm{a}}^{\mathrm{TS} 3} / \mathrm{eV}$ & $E_{\mathrm{r} 3} / \mathrm{eV}$ \\
\hline$[\mathrm{Cu}-\mathrm{O}-\mathrm{Pd}]^{2+}$ & 0.48 & -1.74 \\
{$[\mathrm{Cu}-\mathrm{O}-\mathrm{Pt}]^{2+}$} & 0.05 & -2.39 \\
{$[\mathrm{Cu}-\mathrm{O}-\mathrm{Fe}]^{2+}$} & 0.45 & -3.14 \\
{$[\mathrm{Cu}-\mathrm{O}-\mathrm{Co}]^{2+}$} & 0.02 & -1.22 \\
{$[\mathrm{Cu}-\mathrm{O}-\mathrm{Ni}]^{2+}$} & 0.27 & -2.48 \\
{$[\mathrm{Cu}-\mathrm{O}-\mathrm{Au}]^{2+}$} & 0.01 & -2.20 \\
{$[\mathrm{Cu}-\mathrm{O}-\mathrm{Ag}]^{2+}$} & 0.47 & -0.34 \\
\hline
\end{tabular}

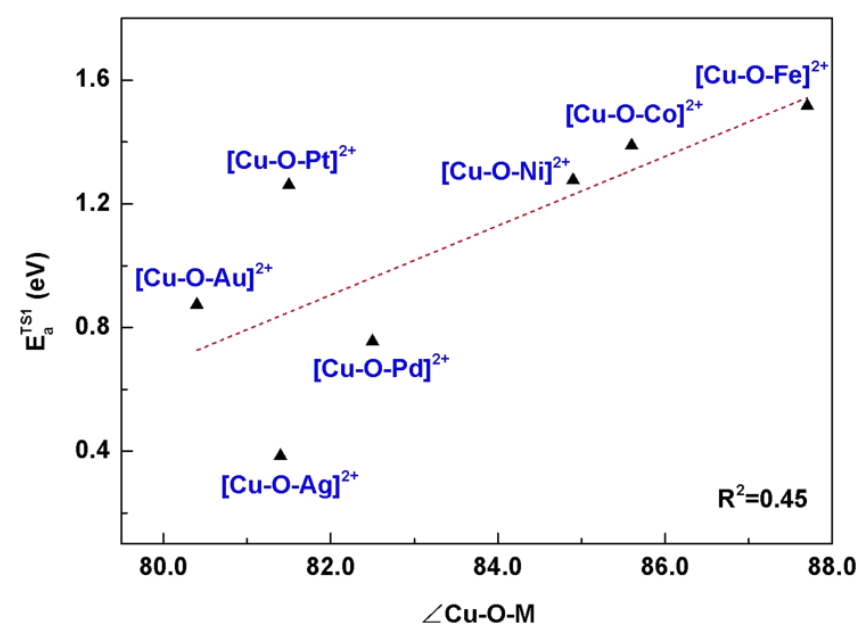

Figure S12. The linear relationship between $\angle \mathrm{Cu}-\mathrm{O}-\mathrm{M}$ and $E_{\mathrm{a}}^{\mathrm{TS} 1}$ of $[\mathrm{Cu}-\mathrm{O}-\mathrm{M}]^{2+}(\mathrm{M}=\mathrm{Pd}, \mathrm{Pt}$, $\mathrm{Fe}, \mathrm{Co}, \mathrm{Ni}, \mathrm{Au}, \mathrm{Ag})$. 